\title{
Non-linear modelling and control of a conveyor-belt grain dryer utilizing neuro-fuzzy systems
}

\begin{abstract}
The grain drying process is characterized by its complex and non-linear nature. As a result, conventional control system design cannot handle this process appropriately. This work presents an intelligent control system for the grain drying process, utilizing the capabilities of the adaptive neuro-fuzzy inference system (ANFIS) to model and control this process. In this context, a laboratory-scale conveyor-belt grain dryer was specifically designed and constructed for this study. Utilizing this dryer, a real-time experiment was conducted to dry paddy (rough rice) grains. Then, the inputï output data collected from this experiment were presented to an ANFIS network to develop a control-oriented dryer model. As the main controller, a simplified proportionalï integralï derivative (PID)-like ANFIS controller is utilized to control the drying process. A real-coded genetic algorithm (GA) is used to train this controller and to find its scaling factors. From the robustness tests and a comparative study with a genetically tuned conventional PID controller, the simplified ANFIS controller has proved its remarkable ability in controlling the grain drying process represented by the developed ANFIS model.
\end{abstract}

Keyword: Conveyor-belt grain dryers; ANFIS modelling and control; Genetic algorithms; Conventional PID controller 